\title{
In vitro co-cultures of Pinus pinaster with Bursaphelenchus xylophilus: a biotechnological approach to study pine wilt disease
}

\author{
Jorge M. S. Faria • Inês Sena • Inês Vieira da Silva • Bruno Ribeiro • \\ Pedro Barbosa $\cdot$ Lia Ascensão $\cdot$ Richard N. Bennett • Manuel Mota • \\ A. Cristina Figueiredo
}

Received: 17 November 2014 / Accepted: 31 January 2015/Published online: 13 February 2015

(c) Springer-Verlag Berlin Heidelberg 2015

\begin{abstract}
Main conclusion Co-cultures of Pinus pinaster with Bursaphelenchus xylophilus were established as a biotechnological tool to evaluate the effect of nematotoxics addition in a host/parasite culture system.
\end{abstract}

The pinewood nematode (PWN), Bursaphelenchus $x y$ lophilus, the causal agent of pine wilt disease (PWD), was detected for the first time in Europe in 1999 spreading throughout the pine forests in Portugal and recently in Spain. Plant in vitro cultures may be a useful experimental system to investigate the plant/nematode relationships in loco, thus avoiding the difficulties of field assays. In this study, Pinus pinaster in vitro cultures were established and compared to in vivo 1 year-old plantlets by analyzing shoot structure and volatiles production. In vitro co-cultures were established with the PWN and the effect of the phytoparasite on in vitro shoot structure, water content and volatiles production was evaluated. In vitro shoots showed similar structure and volatiles production to in vivo maritime pine plantlets. The first macroscopic symptoms of

J. M. S. Faria · I. Sena · I. Vieira da Silva · L. Ascensão ·

A. C. Figueiredo $(\bowtie)$

Universidade de Lisboa, Faculdade de Ciências de Lisboa, DBV,

CESAM, Centro de Biotecnologia Vegetal, C2, Campo Grande,

1749-016 Lisbon, Portugal

e-mail: acsf@fc.ul.pt

J. M. S. Faria

e-mail: jmfaria@fc.ul.pt

I. Sena

e-mail: ines.carvalho.sena@gmail.com

I. Vieira da Silva

e-mail: imvieiradasilva@hotmail.com

L. Ascensão

e-mail: lia.ascensao@fc.ul.pt
PWD were observed about 4 weeks after in vitro co-culture establishment. Nematode population in the culture medium increased and PWNs were detected in gaps of the callus tissue and in cavities developed from the degradation of cambial cells. In terms of volatiles main components, plantlets, $P$. pinaster cultures, and $P$. pinaster with $B$. xylophilus co-cultures were all $\beta$ - and $\alpha$-pinene rich. Cocultures may be an easy-to-handle biotechnological approach to study this pathology, envisioning the understanding of and finding ways to restrain this highly devastating nematode.

Keywords Maritime pine - Monoxenic culture Pinewood nematode $\cdot$ Relative water content $\cdot$ Shoots structure · Volatiles

\section{Abbreviations}

BAP 6-Benzylaminopurine

DAI Days after inoculation

EPPO European and Mediterranean Plant Protection Organization

B. Ribeiro · P. Barbosa · M. Mota

NemaLab/ICAAM, Instituto de Ciências Agrárias e Ambientais Mediterrânicas, Universidade de Évora, Núcleo da Mitra, Ap. 94, 7002-554 Évora, Portugal e-mail: brunoduarteribeiro@gmail.com

P. Barbosa

e-mail: pmbarbosa@yahoo.com

M. Mota

e-mail:mmota@uevora.pt

\section{R. N. Bennett}

Universidade de Trás-os-Montes e Alto Douro, Quinta dos

Prados, Apartado 1013, 5000-911 Vila Real, Portugal

e-mail: rnbennett@utad.pt 
GC Gas chromatography

GC-MS Gas chromatography coupled to mass spectrometry

IBA Indole-3-butyric acid

LM Light microscopy

PAS Periodic acid-Schiff's reagent

PWD Pine wilt disease

PWN Pinewood nematode (Bursaphelenchus xylophilus)

RI Retention index

RWC Relative water content

SEM Scanning electron microscopy

SH Schenk and Hildebrandt medium

SHe Schenk and Hildebrandt elongation medium

SHm Schenk and Hildebrandt multiplication medium $\mathrm{t} \quad$ Trace

\section{Introduction}

The pine wilt disease (PWD) is caused by the pinewood nematode (PWN), Bursaphelenchus xylophilus (Steiner \& Buhrer) Nickle, which is a highly pathogenic, migratory, facultative endoparasite which generally infects some $\mathrm{Pi}$ nus species. In Portugal, maritime pine, Pinus pinaster Aiton, is highly susceptible to infection. In 1999, the nematode was detected in Portugal (Mota et al. 1999) endangering European pine forests and has progressed throughout large areas of the country (Mota and Vieira 2008). In 2010, it was also found in Madeira island (Fonseca et al. 2012), and in 2011 for the first time in Spain (Abelleira et al. 2011). It was classified as an A2 type quarantine pest by the European and Mediterranean Plant Protection Organization (EPPO 2012).

The PWN dispersal and life cycle are dependent on vectors, cerambycid Monochamus spp., that include $M$. alternatus in East Asia, M. saltuarius in Japan, M. carolinensis in North America and M. galloprovincialis, abundant in the Portuguese pine forest (Mota and Vieira 2008, Petersen-Silva et al. 2014). After feeding on the fungus growing on dead or decaying wood (mycophagous phase), the nematodes molt into dispersal "third-stage dauer juvenile", $\mathrm{J}_{\mathrm{III}}$, able to outstand adverse conditions. Gathering around the developing insect, "fourth-stage dauer juvenile" $\left(\mathrm{J}_{\mathrm{IV}}\right)$ enters the tracheal system of the emerging young callow adult through its spiracles. Infection of susceptible Pinus spp. occurs in the dispersal phase when adult beetles transmit the $\mathrm{J}_{\mathrm{IV}}$ to other trees while feeding on young tree branches (Futai 2013). At this stage, PWNs are attracted to pine volatile cues that seem to determine changes in their development, particularly major terpenes ratio (Zhao et al. 2007) and/or $\beta$-myrcene content, as well as internal PWN neutral lipid energy reserves (Stamps and Linit 2001).
Once inside the host plant, the nematodes reproduce and multiply at a very high rate in the resin canals, consuming the epithelial cells (phytophagous phase), thus damaging internal pine structure. As infection progresses, embolized tracheids rapidly enlarge and water potential decreases ultimately leading to abrupt cavitation in the whole xylem area (Umebayashi et al. 2011). At this stage, cavitation effects appear to be promoted by increase in production of terpenes by ethylene cues (Wang et al. 2010).

As the tree very quickly begins displaying the characteristic wilting symptoms, "drying out" and yellowing of the pine needles, the oleoresin exudation decreases and as a consequence nematodes are able to move freely through the dying tree (Ikeda and Oda 1980; Kuroda 2008). Although stem anatomy is thought to be linked to variations in pine susceptibility, for e.g. the arrangement of the resin canals (Kuroda 2004) or lignification of infected pine cell walls (Kusumoto et al. 2014), it is not yet established which anatomy characteristics influence PWN progression. The trees showing intensified wilting and yellowing of the needles may collapse within 1-4 months (EPPO 2012). The decaying trees are hosts to the oviposition of female beetles and the remaining life cycle progresses as described above (Mota and Vieira 2008).

The effect of nematotoxic compounds on this phytoparasite has been well-documented, mainly using direct contact bioassays (Choi et al. 2007; Barbosa et al. 2010, 2012; Andrés et al. 2012; Faria et al. 2013). However, research is commonly performed on the nematode species alone and very seldom on the host-parasite system, not taking into account the cytotoxicity to the plant host or the plant's capability to metabolize or biotransform the nematotoxic active substances.

By co-culturing host and parasite at the same time, simulating the host-pathogen conditions, in vitro culture can be a useful system to study plant/nematode interactions, since it allows (a) eliminating variables due to environmental conditions, (b) having a contaminant-free system, which, by being in a monoxenic culture, excludes the diverse-associated microbiota (Amerson and Mott 1982; Vicente et al. 2012), (c) manipulating single variables, making possible the direct observation of plant/nematode responses in a controlled environment, which is very difficult to achieve in greenhouse or in field conditions, and also (d) attaining more biomass using fewer resources.

The present study aimed at developing a reliable host/pathogen system for PWD phytopathological research. To accomplish this, in vitro $P$. pinaster and in vitro $P$. pinaster/B. xylophilus co-cultures were established. PWN density in the co-culture medium was followed as well as in vitro pine relative water content. Healthy 1 -year-old plantlets, pine in vitro cultures and pine/PWN co-culture 
structure and volatile production were also determined. The present work proposes maritime pine/PWN co-cultures as an adequate biotechnological tool to study the PWD, capable of simulating many conditions of the ex vitro nematode infection.

\section{Materials and methods}

In vitro cultures establishment

\section{Pinus pinaster cultures (shoots)}

Seeds from maritime pine trees grown at Mata Nacional do Escaroupim, Portugal, were washed with running tap water for $5 \mathrm{~min}$, then immersed in a commercial detergent (surfactants: anionic $\geq 15$ and $<30 \%$, non-ionic $\geq 5$ and $<15 \%$, disinfectant: triclosan $0.1 \%$ ) solution (10 drops per $100 \mathrm{ml}$ of distilled water) for $10 \mathrm{~min}$ and dipped in an ultrasonic bath, 5 times for about $1 \mathrm{~min}$ at a time. After rinsing with running tap water, the seeds were surface sterilized by immersion in ethanol $96 \%$, in an ultrasound bath for $10 \mathrm{~min}$, as before. In asepsis, the seeds were rinsed, three times, with ultrapure sterile water, approx. $100 \mathrm{ml}$ each, and the outer seed coat was broken with a mechanical lathe. Pine nuts were hydrated in sterile ultrapure water, stratified at $4{ }^{\circ} \mathrm{C}$ for 2 days and sown in sterile wetted filter paper in covered glass jars. Seedlings were maintained in darkness, at $24 \pm 1{ }^{\circ} \mathrm{C}$, for 1 week and then transferred to a $16 \mathrm{~h}$ light photoperiod [cool fluorescent lamps $\left.\left(32 \mu \mathrm{E} \mathrm{m}^{-2} \mathrm{~s}^{-1}\right)\right]$.

The seedling from one genotype was sectioned and the upper portion (hypocotyl and cotyledon) was maintained on multiplication medium ( $\mathrm{SHm})$, that is, on solid $\mathrm{SH}$ culture medium (Schenk and Hildebrandt 1972) with $30 \mathrm{~g}^{-1}$ sucrose, supplemented with $0.5 \mathrm{mg} \mathrm{l}^{-1}$ 6-benzylaminopurine (BAP) and $0.1 \mathrm{mg} \mathrm{l}^{-1}$ indole-3-butyric acid (IBA). The $\mathrm{pH}$ was adjusted to 5.8 prior to the addition of $0.8 \%(\mathrm{w} / \mathrm{v})$ agar and autoclaved at $121{ }^{\circ} \mathrm{C}$ for $15 \mathrm{~min} . P$. pinaster shoots were maintained in Combiness ${ }^{\circledR}$ (Belgium) microboxes $[9.7 \mathrm{~cm}$ base diameter per $8 \mathrm{~cm}$ height and green filter $(\mathrm{XXL}+)$ on the lid, to facilitate air exchange], in a growth chamber with temperature and photoperiod as above. Under routine culture conditions, every 4 weeks, each shoot cluster was subdivided into 3-4 smaller clusters and transferred to microboxes with $100 \mathrm{ml}$ fresh culture medium.

For shoot elongation, $P$. pinaster shoot masses (7-10 shoots) were transferred to an elongation medium ( $\mathrm{SHe}$ ), that is, to solid SH medium, without growth hormones and with activated charcoal $\left(3 \mathrm{~g} \mathrm{l}^{-1}\right)$, adapted from Tereso et al. (2006). Elongation allowed shoots to be detached from the main mass and individualized. In vitro cultures were maintained as described above and subculture was performed monthly. Elongation rate was followed monthly by measuring individual shoot length, for 32 months. A minimum of 30 in vitro shoots were measured per month. The data were statistically analyzed using Microsoft Excel 2013.

Pinus pinaster with Bursaphelenchus xylophilus cocultures (co-cultures)

Bursaphelenchus xylophilus (isolate BxPt51T, retained at NemaLab and available on request) was obtained as described by Faria et al. (2013). Surface sterilization was performed in aliquots of $500 \mu$, with $3250 \pm 250$ mixedstage PWNs in ultrapure water. In asepsis, nematodes were suspended in a $50 \%$ ethanol/ultrapure sterile water solution $(\mathrm{v} / \mathrm{v})(20 \mathrm{ml})$, for $5 \mathrm{~min}$ in a $20 \mu \mathrm{m}$ mesh sieve, and then washed 5 times in ultrapure sterile water, $20 \mathrm{ml}$ each, resuspended in $1 \mathrm{ml}$ sterile water. PWNs sterilization was tested on potato dextrose agar plates for 4 days at $25^{\circ} \mathrm{C}$. Sterilized PWNs were used for inoculating in vitro $P$. pinaster cultures.

Establishment of co-cultures was initiated by transferring $P$. pinaster shoots, maintained for 5-7 months in $\mathrm{SHe}$, with monthly subculture, to activated charcoal-free solid SHe medium. A $100 \mu \mathrm{l}$ suspension (250 \pm 50 PWNs) was added into a small hole made in the culture medium into which the cut end of each shoot was inserted (Fig. 1). Cultures were maintained as described above. For further subculture, 4-weeks co-culture-grown PWNs were resuspended in $1 \mathrm{ml}$ sterile water and used as described above.

Characterization of in vitro cultures and plantlets

Pinus pinaster cultures and $P$. pinaster with $B$. xylophilus co-cultures were evaluated in terms of structure, relative water (RWC), PWN population growth and volatiles content. For in vitro culture characterization, maritime pine shoots were subcultured to microboxes $[8 \mathrm{~cm}$ base diameter per $6 \mathrm{~cm}$ height and green filter $(\mathrm{XXL}+)$ on the lid] with $20 \mathrm{ml}$ solid SH medium (2 shoots per microbox). Whereas some shoots were kept uninfected, as control, others were infected with sterilized PWN suspension as described above. Sampling was performed before infection (time 0 ) and 1, 2, 7, 28, and 35 days after inoculation (DAI), both for $P$. pinaster shoots and for $P$. pinaster shoots inoculated with $B$. xylophilus. Culture conditions were maintained as described above. Two independent experiments were separately run and four replicates were used in each experiment. All statistical analyses were performed using Microsoft Excel 2013.

For comparison purposes, samples from greenhouse grown 1-year-old $P$. pinaster plantlets were harvested for 

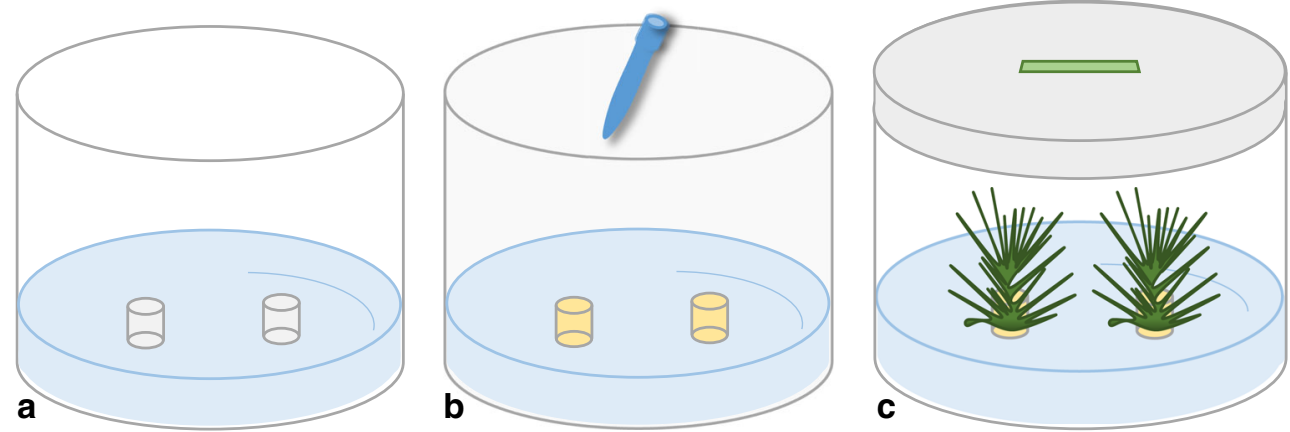

Fig. 1 Schematic representation of $P$. pinaster with B. xylophilus co-cultures establishment. Under asepsis, small holes were made in the culture medium (a), into which a $100 \mu \mathrm{lWNs}$ suspension (250 \pm 50 PWNs) was added (b) together with each pine shoot (c)

structure and volatiles characterization. Plantlets were obtained from a mainland Portuguese nursery field (Alcácer do Sal, from seeds made available from Mata Nacional do Escaroupim, Portugal) maintained under natural $16 \mathrm{~h}$ light photoperiod, with average $30{ }^{\circ} \mathrm{C}$ day $/ 18{ }^{\circ} \mathrm{C}$ night temperature and about $60 \%$ of relative humidity. As above, two independent experiments were separately run and 4 replicates were used in each experiment. All statistical analyses were performed using Microsoft Excel 2013.

\section{Nematode population in the co-culture}

Pinewood nematodes present in the co-culture medium were counted as a measure of nematode population growth over time. Nematodes were counted by sampling $100-\mu 1$ aliquots, three times, from $2 \mathrm{ml}$ used to wash the co-culture medium of each microbox. To rule out nematode feeding on SH culture medium, the PWN population was compared between in vitro cultures with and without maritime pine shoots, at 28 DAI. PWNs were counted under an inverted microscope [Diaphot, Nikon, Japan $(40 \times)$ ].

At 28 DAI, the length and diameter of 30 of each randomly selected PWN females, males and juveniles (J2-J4) were measured using a stage micrometer calibrated eyepiece reticle and compared with that of PWN population grown on Botrytis cinerea. Permanent slides were prepared as described by Ryss (2003).

\section{Shoots and co-cultures relative water content}

Relative water content (RWC) was evaluated at 0, 1, 2, 7, 28 and 35 DAI, through the following formula:

$$
\begin{aligned}
& \text { Relative water content }(\%) \\
& =[(\text { fresh weight }- \text { dry weight }) /(\text { fresh weight })] \times 100
\end{aligned}
$$

In vitro shoot fresh weight determination was performed after carefully rinsing and blotting culture medium excess with filter paper. For dry weight calculation, samples were frozen for $24 \mathrm{~h}$ followed by freeze-drying for 2 days, in an Alpha I-5 (Martin Christ GmbH, Osterode, Germany) apparatus, at 0.1 mbar and $-42{ }^{\circ} \mathrm{C}$.

\section{Plantlets, shoots and co-cultures structure}

In vitro shoots and co-cultures morphology and anatomy were analyzed by scanning electron microscopy (SEM) and light microscopy (LM). Sampling was performed before infection (time 0) and at the 7, 28, and 35 DAI. At each sampling time point, in vitro shoot cross-sections were processed after striping the in vitro pine needles. Samples from greenhouse grown 1-year-old $P$. pinaster plantlets were processed in a similar way.

For SEM, $P$. pinaster shoots and co-culture shoots were fixed with glutaraldehyde $2.5 \%(\mathrm{v} / \mathrm{v})$ in $0.1 \mathrm{M}$ sodium phosphate buffer at $\mathrm{pH}$ 7.2. Samples were kept in fixative under vacuum at room temperature for $20 \mathrm{~min}$, followed by $24-48 \mathrm{~h}$ at $4{ }^{\circ} \mathrm{C}$. The material was then washed in the fixative buffer, dehydrated in a graded ethanol series, and critical point-dried in a Polaron E3500, according to Ascensão et al. (2005). Dried specimens were sputter coated with gold in a Polaron E5350. Observations were carried out on a JEOL T220 scanning electron microscope (JEOL Ltd., Tokyo, Japan) at $15 \mathrm{kV}$.

For LM, P. pinaster shoots and co-culture shoots were fixed as described for SEM, but after the washes in fixative buffer and dehydration through an ethanol series, the material was infiltrated and embedded in Leica historesin ${ }^{\circledR}$ according to Ascensão et al. (2005). To highlight the contrast between the plant tissues and PWNs, longitudinal and cross-sections ( $3 \mu \mathrm{m}$ thick) were stained with periodic acid-Schiff's (PAS) reagent for polysaccharides, counterstained with Toluidine Blue O (Feder and O'Brien 1968) for general histology, and with Coomassie blue stain (Fisher 1968) for proteins. Observations were made with a Leica DM-2500 microscope (Leica Microsystems CMS $\mathrm{GmbH}$, Wetzlar, Germany), images were recorded digitally 
using a Leica DFC-420 camera (Leica Microsystems Ltd., Heerbrugg, Switzerland) and the Leica Application Suite software (version 2.8.1).

\section{Plantlets, shoots and co-cultures volatiles}

Pinus pinaster shoots and co-cultures volatiles were sampled at $0,2,7,14,28,35$ days after subculture and at $1 \mathrm{~h}, 8 \mathrm{~h}$ and 1, 2, 7, 14, 28 and $35 \mathrm{DAI}$, respectively. Isolation was performed by distillation-extraction, for $3 \mathrm{~h}$, using a Likens-Nickerson type apparatus (Likens and Nickerson 1964). Distillation was run at a distillation rate of $3 \mathrm{ml} \mathrm{min}{ }^{-1}$, using in-lab distilled $n$-pentane $(50 \mathrm{ml})$ (Honeywell Riedel-de Haën, Hanover, Germany) as organic solvent. The volatiles recovered in distilled $n$-pentane were concentrated at room temperature under reduced pressure on a rotary evaporator, collected in a vial, and concentrated to a minimum volume, again at room temperature, under nitrogen flux. In vivo pine essential oils were isolated by hydrodistillation for $3 \mathrm{~h}$ using a Clevenger type apparatus according to the European Pharmacopoeia (Council of Europe 2010). Hydrodistillation was run at a distillation rate of $3 \mathrm{ml} \mathrm{min}{ }^{-1}$. The volatile oils were stored at $-20{ }^{\circ} \mathrm{C}$ until analysis. Volatiles were analyzed by gas chromatography (GC), for component quantification, and gas chromatography coupled to mass spectrometry (GC-MS) for component identification, as detailed by Faria et al. (2014).

\section{Results and discussion}

Pinus pinaster cultures establishment

Pinus pinaster in vitro shoots multiplication and elongation growth regulators requirements were optimized as reported by Calixto and Pais (1997) and Álvares et al. (2009). P. pinaster shoots subculture in SHm, induced meristem multiplication along the apical meristem shoot, within 4 weeks after subculture, and lead to the formation of clusters of apical needles buds (shoot clusters) (Fig. 2a). These were detached from the main multiplying shoot and subcultured monthly in the SHm. For shoot elongation, the shoot clusters were transferred from SHm to SHe medium, containing activated charcoal. Activated charcoal acts by adsorbing many organic and inorganic molecules, released from growing explants or from the culture medium. To ensure in vitro culture stability, shoots were transferred from SHm to SHe medium only after approx. 12 months in $\mathrm{SHm}$ culture, with routine subculture. Elongation period resulted in $3 \mathrm{~cm}$ shoots being obtained within about 5 months (Fig. 2b). In this period, maintenance in $\mathrm{SHe}$ induced shoot elongation at rates of about $0.9 \mathrm{~mm} \mathrm{week}^{-1}$ $\left(R^{2}=0.99\right)$, that became very low after 5 months. Shoots with 5-7 months of subculture in SHe were selected for infection with the sterilized PWN.

\section{P. pinaster with B. xylophilus co-cultures establishment}

Within 3-4 weeks after $P$. pinaster inoculation with $B$. $x y$ lophilus, the first external signs of the PWD were observed, with several of the shoot pine needles exhibiting wilting symptoms like chlorosis and drooping when compared to control (Fig. 2cf). These symptoms were detected in the older pine needles and progressed to the younger, towards the shoot apex, 5-6 weeks after infection the shoot was entirely brown and necrotic. In vitro infection showed to be similar to in vivo PWD phenotype as a similar symptomatology was observed in trees infected by the pine wilt disease, as reported by Kuroda et al. (1988) for P. thunbergii and P. densiflora saplings. In these species, the first symptom observed was the sudden browning of older needles that spread to younger needles accompanied by wilting and followed by host death within 1-2 months after inoculation. Symptom development in these pine species varies depending on tree age, as younger seedlings appeared to develop symptoms more rapidly than older saplings and older trees (Kuroda et al. 2007). Studying the pathogenicity of aseptic PWNs in in vitro $P$. densiflora, Zhu et al. (2012) obtained PWD symptomatology at 20 days of infection with a 250 nematode initial inoculum. Wilting and browning were observed with infection performed on the upper portion of the micro-cutting. The authors proved that aseptic PWNs maintain their pathogenicity and infection progressed to micro-cutting death. Being immature tissue, the in vitro shoots may be affected more promptly. This observation is in agreement with that herewith reported. Overall co-cultures showed similar symptoms to maritime pine under natural infection conditions.

There is an ongoing debate on the role of bacterial communities associated to the PWN on PWD. Population variations of bacterial communities generally follow those of PWN progression (Xie and Zhao 2008; Roriz et al. 2011; Nascimento et al. 2014) and evidence as pointed towards being potential triggers for disease symptomatology (Han et al. 2003; Vicente et al. 2012) and even promoters of PWN reproduction and fecundity (Zhao et al. 2006).

The data obtained in the present work for in vitro grown pine tissue, support Zhu et al. (2012), in that PWD symptomatology does not seem to be solely dependent on associated microorganism communities.

The morphometric parameters of PWN co-cultured with $P$. pinaster showed adult male body greatest diameter/length $15.6 \pm 0.6 \mu \mathrm{m} / 685.7 \pm 17.7 \mu \mathrm{m}$, female $20.1 \pm 0.5 \mu \mathrm{m} /$ $760.5 \pm 26.4 \mu \mathrm{m}$ and juveniles (J2-J4) $12.4 \pm 0.5 \mu \mathrm{m} /$ $421.9 \pm 17.7 \mu \mathrm{m}$, were slightly smaller than those from labgrown PWN in Botrytis cinerea: adult male $19.5 \pm 0.4 \mu \mathrm{m} /$ $837.7 \pm 12.2 \mu \mathrm{m}$, female $21.1 \pm 0.3 \mu \mathrm{m} / 896.1 \pm 14.8 \mu \mathrm{m}$ and juveniles $15.7 \pm 0.5 \mu \mathrm{m} / 555.3 \pm 21.8 \mu \mathrm{m}$. The 

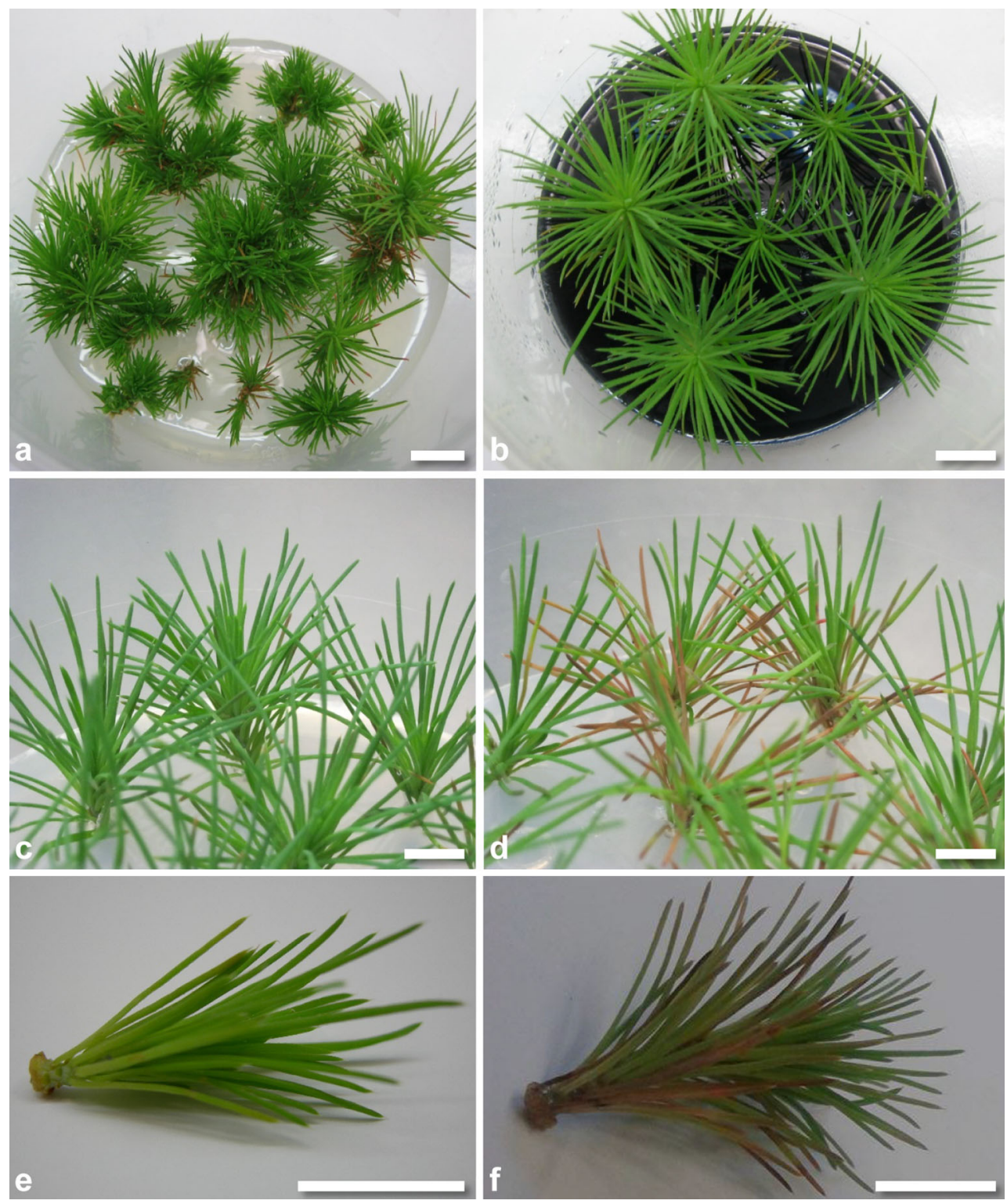

Fig. 2 Pinus pinaster shoots under routine culture conditions grown in $\mathrm{SH}$ multiplication medium (SHm) (a) and in elongation medium (SHe) (b), with monthly subculturing. c-f Details of $P$. pinaster shoots $(\mathbf{c}, \mathbf{e})$ and of $P$. pinaster with Bursaphelenchus xylophilus co-

morphometric values (body length and greatest diameter) obtained in the present study for phytophagous PWNs are in accordance with those obtained by Penas et al. (2008) and Fonseca et al. (2008). The tendency for smaller individuals in PWNs obtained from the field, from naturally infected $P$. pinaster trees when compared with lab-grown mycophagous PWNs was also recorded by Penas et al. (2008).

Shoots and co-cultures relative water content and PWN density in co-culture medium

Being pine needle wilting one of the symptoms of nematode infection, $P$. pinaster co-cultures shoots relative culture 4 weeks after infection $(\mathbf{d}, \mathbf{f})$. Note, in $\mathbf{d}$ and $\mathbf{f}$, that shoot needles exhibited wilting, that is, a yellow-brownish color due to chlorosis and drooping. Scale bar $1 \mathrm{~cm}$

water content (RWC) was assessed at 0, 1, 2, 7, 28 and 35 DAI, as a measure of PWN infection mechanism. The RWC of in vitro pine shoots varied between 72 and $85 \%$, not showing substantial variations (Fig. 3). Although other symptoms of PWD such as needle chlorosis were visible at latter stages of growth, the fact that no major needle desiccation was observed, during the period evaluated, may reflect the growth under in vitro-specific conditions that prevents main water loss by evaporation. Under natural conditions, interruption of the water column leads to the process of pine cavitation which diminishes water content. PWD-derived cavitation leads to discoloration in the pine needles and a decrease in 
photosynthesis (Kuroda 2008) and is responsible for pine needle desiccation.

Nematode population in the co-culture medium was measured 1, 2, 7, 28 and 35 DAI. PWN inoculum of $250 \pm 50$ nematodes per shoot increased, having doubled by the end of the first week (Fig. 3). After 4 weeks in culture, PWNs amounted $1900 \pm 204$ nematodes per shoot, while solely in SH medium remained at $242 \pm 60$. An approx. $8 \times$ increase indicates that nematodes reproduced and completed their life cycle as in natural conditions, consuming shoot tissue to increase population numbers. PWN population numbers continued to increase and at the end of the 5th week reached $4340 \pm 504$ PWN per shoot. Given optimal conditions, PWN life cycle can be completed in 4 days, which is very rapid when compared with other Bursaphelenchus species (Futai 2013). In the present study, PWN population doubling time was approximately 1 week, which indicates that even though feeding may have occurred on the shoot basal zone in contact with culture medium, PWN population increase was still considerable.

\section{$P$. pinaster plantlets, shoots and co-cultures structure}

Maritime pine in vitro shoots showed the typical structure from young pine plantlets - a pith with a medullar parenchyma, a vascular ring with axial and radial resin ducts, a starch-rich cortical parenchyma with several tannin-containing cells and axial resin ducts (Figs. 4a, c, 5b). Recently developed $P$. pinaster in vitro grown shoots

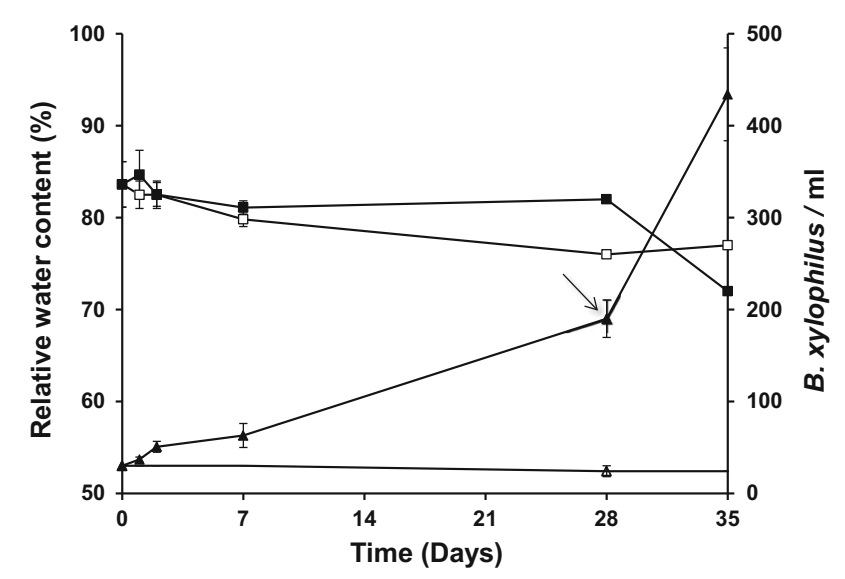

Fig. 3 Relative water content (\%) of in vitro P. pinaster shoots (open squares) and of $P$. pinaster shoots with PWN co-culture (filled squares). Nematode population density in the microbox culture medium (filled triangles) at the different time points of the timecourse study and at 0 and 28 days without pine shoots (open triangles). Two shoots were maintained per container in $20 \mathrm{ml}$ of solid culture medium. Arrow time points when, macroscopically, pine needles started to exhibit wilting (drooping and a yellow-brownish color due to chlorosis) showed ducts with a very narrow lumen. At the cut end of the shoots, resulting of the separation from the parent shoots clusters, and facing the culture medium, a callus tissue formed, characterized by an unorganized mass of loosely arranged parenchyma cells (Fig. 2e, f). In general, depending on the plant material, medium composition and environmental conditions during culture period, callus growth characteristics may be variable in the extent and type of differentiation.

Callus tissue of the pine in vitro shoot facing the culture medium showed clusters of cells giving rise to meristematic zones (Figs. 4b, 5a). This basal shoot zone in contact with the culture medium is a sink for endogenous phytohormones and lesion-derived stress compounds that stimulate tissue dedifferentiation and formation of cell meristematic centers (Washer et al. 1977; Aitken-Christie et al. 1985). These centers with starch-rich cells continued to grow throughout the culture period surrounding primordial tracheary elements (Fig. 4b, arrows). The growth and development of the meristematic centers was accompanied by the formation of fissures in the parenchyma tissue due to movement of cell masses.

One week after co-culture establishment of $P$. pinaster with PWN, numerous nematodes were found within callus parenchyma tissue gaps (Figs. 4e, 5c, d). Parasite feeding may have stimulated callus tissue development since, in addition to mechanical injury, callus tissue may be produced as a response to an invading organism. Four weeks after infection, at less than one centimetre above the cut end of the shoots, vascular bundles were already present and nematodes were observed in cavities formed in the cambium between the xylem and phloem (Fig. 4d, arrow). Above this shoot basal region no more nematodes were found, although shoots exhibited the typical anatomy of a Pinus species. Fully-developed resin canals were yet scarce and presented narrow lumens, being only frequent secretory ducts in early ontogenic stages.

Iwahori and Futai (1990) analyzed calli obtained from several susceptible and resistant pine species $(P$. densiflora, $P$. thunbergii, $P$. massoniana, $P$. thunbergii $\times P$ massoniana, P. taeda) as well as Nicotiana tabacum and Medicago sativa as a method to obtain clean PWN populations. Although high PWN growth rates were detected, probably due to an easy access to food source, callus tissue culture unorganized nature was not faithful to in vivo pine characteristics. Thin-walled metabolically very active cambial cells may serve for nematode feeding during the infection process. In fact, it is now well-known that nematode secretions are rich in cell wall degrading enzymes such as the $\beta-1,4-$ and $\beta$-1,3-glucanases, pectate lyase and also expansins and cellulose-binding proteins (Haegeman et al. 2012; Shinya et al. 2013a, b). In the current study, nematode secretions may have influenced greatly in vitro shoot PWD symptom 


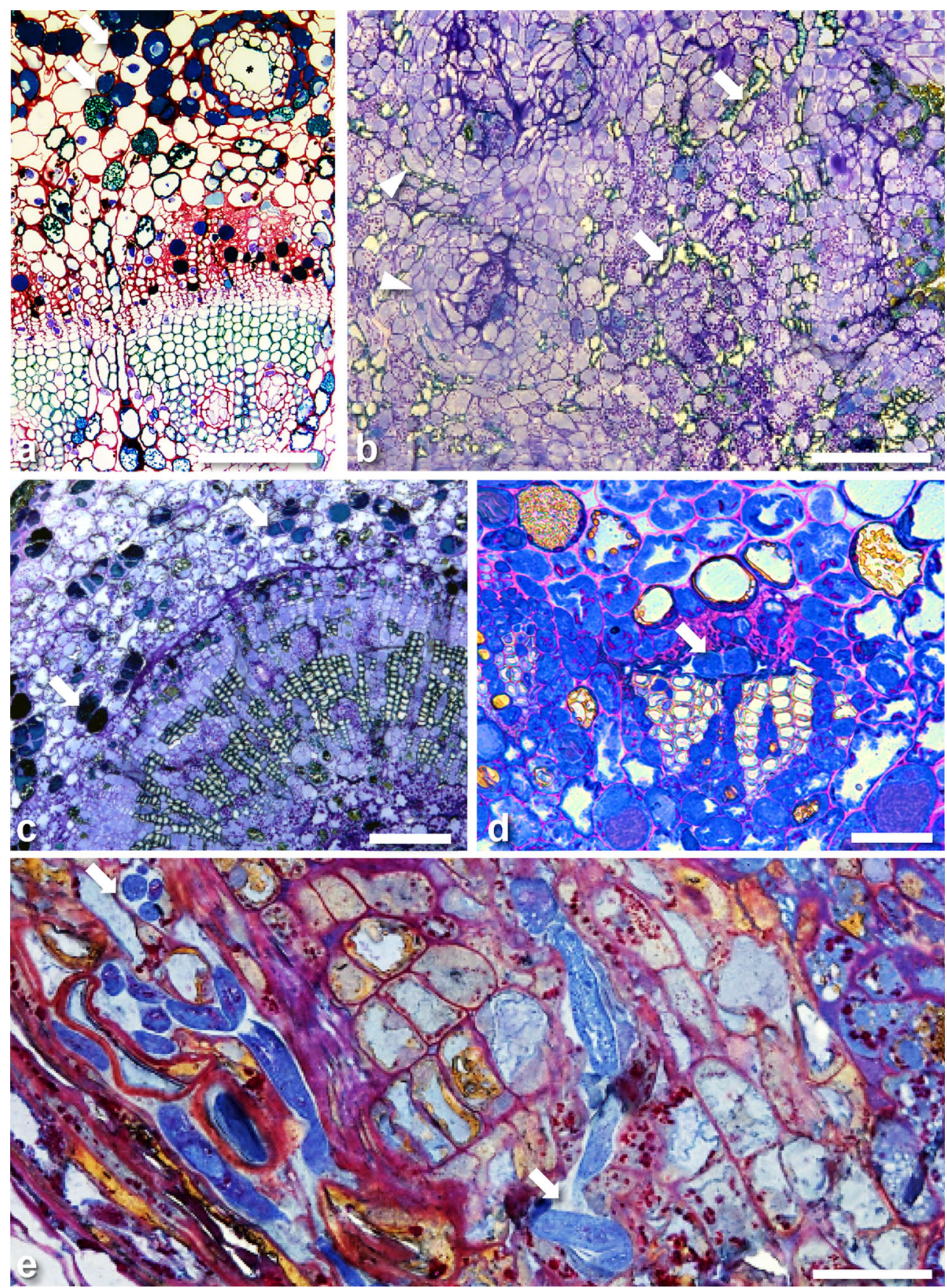

Fig. 4 Light micrographs of historesin sections of shoots from 1-year-old Pinus pinaster seedlings (a), from in vitro shoot cultures (b, c), and from shoot co-cultures with Bursaphelenchus xylophilus (d, e). a Cross-section showing the characteristic anatomy of a pine shoot. Note the presence of several tanniniferous cells (arrows) in the cortical parenchyma. Resin ducts (asterisks) are clearly seen in the cortex and xylem. b Callus tissue, in the zone facing the culture medium, showing the dedifferentiation centers (arrowheads) and

development, namely, macroscopically, pine shoot wilting (desiccation, chlorosis and drooping). Plant tissue degeneration was noticeable as nematode population increased, probably not solely due to nematodes feeding, as well as to tracheary elements (arrows). c A vascular ring, surrounding the pith and showing tanniniferous cells (arrows), is observed in shoot crosssections some millimeters above the culture medium. d, e Nematodes were found in cavities developed in the vascular bundles between the xylem and the phloem (d, arrow) and in gaps formed in the callus tissue during the dedifferentiation process (e). Scale bars $200 \mu \mathrm{m}(\mathbf{a})$, $50 \mu \mathrm{m}(\mathbf{b}), 100 \mu \mathrm{m}(\mathbf{c})$ and $40 \mu \mathrm{m}(\mathbf{d}, \mathbf{e})$

parasite secretions. In fact, Melakeberhan and Webster (1992) analyzing the energy requirements of the PWN in $P$. sylvestris, concluded that food consumption is not a significant factor in the cause of pine death. 


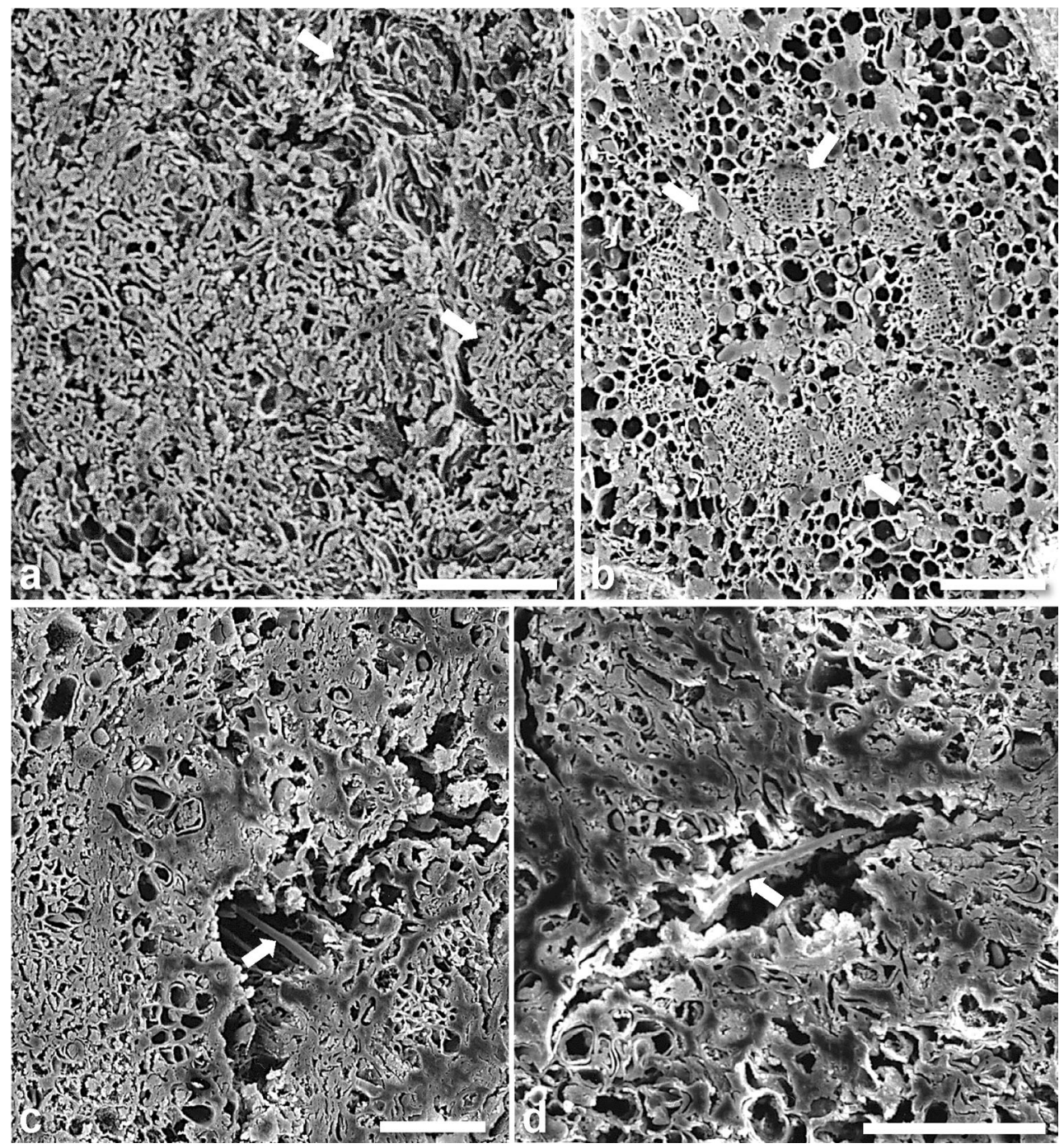

Fig. 5 Scanning electron microscopy micrographs from cross-sections of Pinus pinaster shoots cultures $(\mathbf{a}, \mathbf{b})$ and from $P$. pinaster shoots in co-culture with Bursaphelenchus xylophilus $(\mathbf{c}, \mathbf{d})$. a Several dedifferentiation centers (arrows) are observed in the callus tissue

\section{$P$. pinaster plantlets, shoots and co-cultures volatiles}

Volatiles isolated from in vitro grown $P$. pinaster cultures and $P$. pinaster with $B$. xylophilus co-cultures were compared with those isolated from one-year-old plantlets. Although Table 1 reports only the isolated volatiles main components $(\geq 1 \%)$, they were all fully chemically characterized, in a total of 80 compounds for the plantlets volatiles, 46 for $P$. pinaster cultures and for $P$. pinaster with B. xylophilus co-cultures.

Plantlets volatiles were $\beta$ - and $\alpha$-pinene rich (47 and $28 \%$, respectively) (Table 1 ). Likewise, in vitro pine cultures were also $\beta$ - and $\alpha$-pinene rich $(38-47 \%$ and 24-33\%, respectively), although the relative importance of several compounds differed between plantlets and pine facing the culture medium. b A nearly continuous vascular ring (arrows) is clearly seen in the shoot some millimeters above the culture medium. c, d Nematodes (arrows) are found in callus tissue gaps forming during the dedifferentiation process. Scale bars $100 \mu \mathrm{m}$

cultures volatiles. Whereas $\alpha$-terpineol (7\%) and bornyl acetate $(4 \%)$ were the third- and fourth- plantlets volatiles main components, they were always $<2$ and $<0.5 \%$, respectively, in the pine shoots volatiles. Conversely, germacrene D (3-9 \%), an unidentified compound (UI B Ppi, $4-7 \%)$ and $\beta$-caryophyllene $(2-5 \%)$ that attained relatively high percentages in the pine shoots volatiles, were either $<2 \%$ ( $\beta$-caryophyllene) or in trace amounts (germacrene D and UI B Ppi) (Table 1).

Co-culture of $P$. pinaster with B. xylophilus did not alter substantially the volatile composition compared to pine cultures volatiles. Again $\beta$ - and $\alpha$-pinene dominated the cocultures volatiles (36-47\% and 24-32\%, respectively), followed by germacrene D (3-7 \%), UI B Ppi (4-8 \%) and $\beta$-caryophyllene (1-5\%). 
Table 1 Percentage composition of the volatiles $(>1 \%)$ isolated from $P$. pinaster 1-year-old plantlets aerial parts (plantlets), from in vitro grown $P$. pinaster shoots at $0,2,7,14,28$ and 35 days after subculture (shoots) and from $P$. pinaster/PWN co-cultures at $1 \mathrm{~h}, 8 \mathrm{~h}$ and $1,2,7,14,28$ and 35 days after infection (co-cultures)

\begin{tabular}{|c|c|c|c|c|c|c|c|c|c|c|c|c|c|c|c|c|}
\hline \multirow[t]{2}{*}{ Components $(>1 \%)$} & \multirow[t]{2}{*}{ RI } & \multirow[t]{2}{*}{ Plantlets } & \multicolumn{6}{|c|}{ Shoots } & \multicolumn{8}{|c|}{ Co-cultures } \\
\hline & & & 0 & 2 & 7 & 14 & 28 & 35 & $1 \mathrm{~h}$ & $8 \mathrm{~h}$ & 1 & 2 & 7 & 14 & 28 & 35 \\
\hline$\alpha$-Pinene & 930 & 27.9 & 23.7 & 25.5 & 24.4 & 27.1 & 31.5 & 32.8 & 30.9 & 28.9 & 24.2 & 24.5 & 25.4 & 27.0 & 31.6 & 30.5 \\
\hline Camphene & 938 & 2.2 & 0.2 & 0.2 & 0.5 & 0.6 & 0.6 & 0.5 & 0.5 & 0.5 & 0.3 & 0.4 & 0.6 & 0.6 & 0.5 & 0.5 \\
\hline$\beta$-Pinene & 963 & 46.6 & 37.7 & 38.0 & 42.4 & 43.9 & 46.8 & 43.8 & 44.4 & 38.6 & 36.3 & 38.9 & 39.7 & 42.3 & 47.3 & 42.3 \\
\hline$\beta$-Myrcene & 975 & 0.4 & 1.1 & 1.1 & 1.5 & 1.2 & 1.1 & 1.2 & 1.2 & 0.9 & 2.1 & 1.1 & 1.1 & 2.0 & 1.1 & 1.1 \\
\hline$\beta$-Phellandrene & 1005 & 0.5 & 1.1 & 1.2 & 1.2 & 1.3 & 1.3 & 1.2 & 1.2 & 1.0 & 0.8 & 1.1 & 1.1 & 1.2 & 1.2 & 1.2 \\
\hline Limonene & 1009 & 2.7 & 1.0 & 1.0 & 1.1 & 1.2 & 1.2 & 1.4 & 1.1 & 0.9 & 0.5 & 0.9 & 1.2 & 1.1 & 1.4 & 1.6 \\
\hline Terpinolene & 1064 & 1.4 & 0.1 & 0.7 & 0.4 & 0.5 & 0.6 & 0.5 & 0.2 & 0.3 & 0.2 & 0.3 & 0.3 & 0.3 & 0.7 & 1.0 \\
\hline$\alpha$-Terpineol & 1159 & 7.1 & 0.2 & 0.3 & 1.5 & 1.7 & 1.6 & 1.5 & 0.5 & 0.8 & 0.4 & 1.2 & 1.3 & 1.5 & 2.0 & 4.0 \\
\hline Bornyl acetate & 1265 & 3.6 & 0.1 & 0.1 & 0.3 & 0.4 & 0.3 & 0.4 & 0.1 & 0.3 & 0.2 & 0.2 & 0.2 & 0.3 & $\mathrm{t}$ & $\mathrm{t}$ \\
\hline$\alpha$-Copaene & 1375 & $\mathrm{t}$ & $\mathrm{t}$ & 1.3 & 0.1 & 0.2 & 0.1 & $\mathrm{t}$ & 0.1 & 0.2 & 0.1 & 0.2 & 0.1 & 0.2 & $\mathrm{t}$ & $\mathrm{t}$ \\
\hline$\beta$-Caryophyllene & 1414 & 1.4 & 4.6 & 3.7 & 2.9 & 2.1 & 1.5 & 2.0 & 3.2 & 3.1 & 4.6 & 3.4 & 3.2 & 2.5 & 1.3 & 1.7 \\
\hline$\alpha$-Humulene & 1447 & 0.2 & 0.3 & 1.1 & 0.2 & 0.2 & 0.2 & 0.4 & 0.4 & 0.5 & 0.3 & 0.2 & 0.9 & 0.4 & $\mathrm{t}$ & $\mathrm{t}$ \\
\hline $\begin{array}{l}\text { Phenyl ethyl 2-methyl } \\
\text { butanoate }\end{array}$ & 1467 & 1.7 & 0.2 & 0.1 & 0.3 & 0.4 & 0.4 & 0.5 & 0.2 & 0.3 & 0.2 & 0.2 & 0.5 & 0.5 & 0.2 & 0.2 \\
\hline Phenyl ethyl isovalerate & 1468 & $\mathrm{t}$ & 0.4 & 0.9 & 1.1 & 1.0 & 1.3 & 1.4 & 0.7 & 0.8 & 0.4 & 0.6 & 1.1 & 1.3 & 1.7 & 1.5 \\
\hline Germacrene D & 1474 & $\mathrm{t}$ & 8.8 & 5.3 & 4.4 & 3.6 & 3.3 & 3.2 & 5.0 & 5.1 & 6.9 & 6.8 & 4.5 & 3.1 & 2.6 & 2.9 \\
\hline$\gamma$-Cadinene & 1500 & 0.1 & 0.4 & 2.7 & 1.2 & 0.4 & 0.2 & 0.3 & 0.3 & 1.9 & 1.2 & 1.2 & 1.5 & 0.6 & 1.0 & 1.0 \\
\hline$\delta$-Cadinene & 1505 & 0.1 & 0.7 & 0.1 & 0.9 & 1.5 & 1.0 & 1.1 & 1.6 & 0.5 & 0.5 & 0.8 & 1.0 & 1.8 & $\mathrm{t}$ & $\mathrm{t}$ \\
\hline$\beta$-Caryophyllene oxide & 1561 & 0.2 & 0.3 & 0.3 & 0.3 & 0.2 & $\mathrm{t}$ & 0.1 & 0.2 & 0.2 & 0.6 & 0.9 & 1.0 & 0.2 & $\mathrm{t}$ & $\mathrm{t}$ \\
\hline$\alpha$-Cadinol & 1626 & $\mathrm{t}$ & 0.9 & 0.1 & 0.2 & 0.2 & 0.2 & $\mathrm{t}$ & 0.2 & 0.4 & 0.5 & 1.3 & 0.4 & 0.4 & $\mathrm{t}$ & $\mathrm{t}$ \\
\hline Palmitic acid & 1908 & $\mathrm{t}$ & 2.1 & 2.2 & 0.9 & 0.5 & 0.2 & 0.2 & 0.5 & 1.0 & 1.4 & 2.0 & 0.7 & 0.7 & 0.8 & 1.7 \\
\hline UI B Ppi ${ }^{\mathrm{a}}$ & 2309 & $\mathrm{t}$ & 4.5 & 6.0 & 7.2 & 5.6 & 4.6 & 3.6 & 3.9 & 5.5 & 4.6 & 6.5 & 7.8 & 4.2 & 5.0 & 3.7 \\
\hline$\%$ Identification & & 99.8 & 85.7 & 88.1 & 87.9 & 90.0 & 94.5 & 94.1 & 93.2 & 88.6 & 84.1 & 87.8 & 87.7 & 90.3 & 93.2 & 91.0 \\
\hline \multicolumn{17}{|l|}{ Grouped components } \\
\hline Monoterpene hydrocarbons & & 82.6 & 65.2 & 68.0 & 71.6 & 75.7 & 83.4 & 81.5 & 79.6 & 71.1 & 64.6 & 67.4 & 69.7 & 74.6 & 83.7 & 78.0 \\
\hline $\begin{array}{l}\text { Oxygen-containing } \\
\text { monoterpenes }\end{array}$ & & 12.9 & 0.3 & 0.6 & 2.1 & 2.5 & 2.0 & 1.9 & 0.8 & 1.2 & 0.7 & 1.6 & 1.6 & 2.1 & 2.0 & 4.0 \\
\hline Sesquiterpene hydrocarbons & & 1.8 & 15.4 & 14.9 & 10.3 & 8.7 & 6.7 & 7.3 & 10.7 & 12.1 & 14.3 & 13.1 & 12.0 & 9.4 & 4.9 & 5.5 \\
\hline $\begin{array}{l}\text { Oxygen-containing } \\
\text { sesquiterpenes }\end{array}$ & & 0.3 & 2.0 & 1.4 & 1.3 & 0.9 & 0.4 & 1.0 & 0.7 & 1.7 & 2.1 & 2.7 & 2.0 & 1.4 & $\mathrm{t}$ & 0.3 \\
\hline $\begin{array}{l}\text { Oxygen-containing } \\
\text { diterpenes }\end{array}$ & & 0.1 & & & & & & & & & & & & & & \\
\hline Phenylpropanoids & & 0.4 & & & & & & & & & & & & & & \\
\hline Fatty acids & & $\mathrm{t}$ & 2.1 & 2.2 & 0.9 & 0.5 & 0.2 & 0.2 & 0.5 & 1.0 & 1.4 & 2.0 & 0.7 & 0.7 & 0.8 & 1.7 \\
\hline Others & & 1.7 & 0.7 & 1.1 & 1.8 & 1.8 & 1.8 & 2.2 & 1.0 & 1.6 & 1.0 & 1.2 & 1.8 & 2.2 & 1.9 & 1.7 \\
\hline
\end{tabular}

$R I$ in-lab calculated retention index relative to $\mathrm{C}_{9}-\mathrm{C}_{24} n$-alkanes on the DB-1 column, $t$ trace $(<0.05 \%)$

${ }^{a}$ Unidentified compound detected on Pinus pinaster and on in vitro cultures and co-cultures (standard deviation $<5 \%$ )

Lima et al. (2010) characterized the volatiles from 2-year-old uninoculated healthy $P$. pinaster plants (HP) and from mechanically wounded uninoculated $(\mathrm{C})$ and inoculated (In) individuals. As in the present study, Lima et al. (2010) did not find relevant qualitative and quantitative differences between $\mathrm{HP}, \mathrm{C}$ and In isolated volatiles.
PWN shows chemotaxis to volatile terpenes, altering its behavior due to different volatile cues (Futai 2013; Zhao et al. 2014). The terpenes $\alpha$-pinene, $\beta$-pinene and longifolene appear to be decisive in a fundamental step of the nematode life cycle. Zhao et al. (2007, 2014) showed that different ratios of these terpenes, observed in the host species $P$. massoniana and released by larval vector 
attracted different nematode juvenile stages, and may be the cue to altering from de propagative to the dispersal form. Pine volatile response to inoculation with PWN was analyzed in six-year-old $P$. thunbergii, by Kuroda et al. (1991). In that study, volatile production was enhanced by nematode introduction, associated to the beginning of desiccation; the total volatile terpenes (e.g. $\alpha$-pinene, $\beta$ pinene, $\beta$-myrcene, longifolene) showed a higher concentration when compared with those of healthy trees. Takeuchi et al. (2006) also recorded high emissions of terpenes, like $\alpha$-pinene, while profiling the volatiles of infected $P$. thunbergii. This increase in volatile emissions not only attracts the vector beetle species but appears to contribute to the wilting of the tree, by weakening the tensile strength of the sap, promoting embolism in the tracheids (Kuroda 1991).

Although the present study, using in vitro cultures and cocultures, supported earlier observations with plantlets, that showed no major qualitative differences between the volatiles from healthy and from inoculated plants, further studies on the chemical cues that promote nematode attraction would be relevant. $P$. pinaster in vitro cultures and $P$. pinaster with PWN in vitro co-cultures established and characterized in the present study may constitute a complementary biotechnological tool to investigate not only these chemical cues, but also host and parasite response to nematotoxics.

Author contribution JMSF conceived, designed research, conducted the experiment and wrote the manuscript. IS, IVS and BR conducted the experiment. PB supplied $B$. xylophilus initial inoculum and reviewed the manuscript. LA, RB, MM and ACF supervised the work and reviewed the manuscript. All the authors read and approved the manuscript.

Acknowledgments The authors would like to thank Dr. Célia Miguel (Forest Biotechnology, Instituto de Tecnologia Química e Biológica) for her technical advice, Eng. João Sanches (Mata Experimental do Escaroupim, Instituto da Conservação da Natureza e das Florestas) for supplying maritime pine seeds. M. Mota was partially supported by the EC 7th Framework project REPHRAME KBBE.2010.1.4-09. P. Barbosa and M. Mota also supported by Fundação para a Ciência e a Tecnologia (FCT) under PEst-C/AGR/ UI0115/2011 and PEst-OE/AGR/UI0115/2014. Jorge Faria is grateful to FCT for the PhD grant SFRH/BD/43738/2008. This study was partially funded by FCT, under Pest-OE/EQB/LA0023/2011 and research contract PTDC/AGR-CFL/117026/2010.

Conflict of interest The authors declare that they have no conflict of interest.

\section{References}

Abelleira A, Picoaga A, Mansilla JP, Aguin O (2011) Detection of Bursaphelenchus xylophilus, causal agent of pine wilt disease on Pinus pinaster in northwestern Spain. Plant Dis 95:776
Aitken-Christie J, Singh AP, Horgan KJ, Thorpe TA (1985) Explant developmental state and shoot formation in Pinus radiata cotyledons. Bot Gaz 146:196-203

Álvares JM, Majada J, Ordás RJ (2009) An improved micropropagation protocol for maritime pine (Pinus pinaster Ait.) isolated cotyledons. Forestry 82:175-184

Amerson HV, Mott RL (1982) Phytopathology and tissue culture alliances. In: Bonga JM, Durzan DJ (eds) Tissue culture in forestry. Forestry sciences, vol 5. Martinus Nijhoff/Dr W. Junk Publishers, The Hague, pp 208-230

Andrés MF, Gonzáles-Coloma A, Sanz J, Burillo J, Sainz P (2012) Nematicidal activity of essential oils: a review. Phytochem Rev 11:371-390

Ascensão L, Francisco A, Cotrim H, Pais MS (2005) Comparative structure of the labellum in Ophrys fusca and $O$. lutea (Orchidaceae). Am J Bot 92:1059-1067

Barbosa P, Lima AS, Vieira P, Dias LS, Tinoco MT, Barroso JG, Pedro LG, Figueiredo AC, Mota M (2010) Nematicidal activity of essential oils and volatiles derived from Portuguese aromatic flora against the pinewood nematode, Bursaphelenchus $x y$ lophilus. J Nematol 42:8-16

Barbosa P, Faria JMS, Mendes MD, Dias LS, Tinoco MT, Barroso JG, Pedro LG, Figueiredo AC, Mota M (2012) Bioassays against pinewood nematode: assessment of a suitable dilution agent and screening for bioactive essential oils. Molecules 17:12312-12329

Calixto F, Pais MS (1997) Adventitious shoot formation and plant regeneration from Pinus pinaster Sol. ex Aiton. In Vitro Cell Dev Biol Plant 33:119-124

Choi IH, Park JY, Shin SC, Kim J, Park IK (2007) Nematicidal activity of medicinal plant essential oils against the pinewood nematode (Bursaphelenchus xylophilus). Appl Entomol Zool 42:397-401

Council of Europe (2010) European Directorate for the Quality of Medicines. In: European Pharmacopoeia, 7th edn. Strasbourg, France, $\mathrm{p} 241$

EPPO (2012) Data sheets on quarantine pests: Bursaphelenchus xylophilus. http://www.eppo.int/QUARANTINE/nematodes/Bursa phelenchus_xylophilus/BURSXY_ds.pdf. Accessed 15 Sep 2014

Faria JMS, Barbosa P, Bennett RN, Mota M, Figueiredo AC (2013) Bioactivity against Bursaphelenchus xylophilus: nematotoxics from essential oils, essential oils fractions and decoction waters. Phytochemistry 94:220-228

Faria JMS, Sena I, Maleita CM, Vieira da Silva I, Ascensão L, Abrantes I, Bennett RN, Mota M, Figueiredo AC (2014) In vitro co-culture of Solanum tuberosum hairy roots with Meloidogyne chitwoodi: structure, growth and production of volatiles. Plant Cell Tissue Organ Cult 118:519-530

Feder N, O'Brien TP (1968) Plant microtechnique: some principals and new methods. Am J Bot 55:123-142

Fisher DB (1968) Protein staining of ribboned Epon sections for light microscopy. Histochemie 16:92-96

Fonseca L, Vieira dos Santos MC, Santos MNSA, Curtis RHC, Abrantes I (2008) Morpho-biometrical characterisation of Portuguese Bursaphelenchus xylophilus isolates with mucronate, digitate or round tailed females. Phytopathol Mediterr 47:223-233

Fonseca L, Cardoso JMS, Lopes A, Pestana M, Abreu F, Nunes N, Mota M, Abrantes I (2012) The pinewood nematode, Bursaphelenchus xylophilus, in Madeira Island. Helminthologia 49:96-103

Futai K (2013) Pine wood nematode, Bursaphelenchus xylophilus. Annu Rev Phytopathol 51:5.1-5.23

Haegeman A, Mantelin S, Jones JT, Gheysen G (2012) Functional roles of effectors of plant-parasitic nematodes. Gene 492:19-31

Han ZM, Hong YD, Zhao BG (2003) A study on pathogenicity of bacteria carried by pine wood nematodes. J Phytopathol 151:683-689 
Ikeda T, Oda K (1980) The occurrence of attractiveness for Monochamus alternatus Hope (Coleoptera: Cerambycidae) in nematode-infected pine trees. J Jpn For Soc 62:432-434

Iwahori H, Futai K (1990) Propagation and effects of the pinewood nematode on calli of various plants. Jpn J Nematol 20:25-30

Kuroda K (1991) Mechanism of cavitation development in the pine wilt disease. Eur J For Pathol 21:82-89

Kuroda K (2004) Inhibiting factors of symptom development in several Japanese red pine (Pinus densiflora) families selected as resistant to pine wilt. J For Res 9:217-224

Kuroda K (2008) Physiological incidences related to symptom development and wilting mechanism. In: Zhao BG, Futai K, Sutherland JR, Takeuchi Y (eds) Pine wilt disease. Springer, Heidelberg, pp 201-260

Kuroda K, Yamada T, Mineo K, Tamura H (1988) Effects of cavitation on the development of pine wilt disease caused by Bursaphelenchus xylophilus. Ann Phytopathol Soc Jpn 54:606-615

Kuroda K, Yamada T, Ito S (1991) Development of the pinewilt disease in Pinus densiflora from the stand point of water conduction. J Jpn For Soc 73:69-72

Kuroda K, Ohira M, Okamura M, Fujisawa Y (2007) Migration and population growth of the pine wood nematode (Bursaphelenchus xylophilus) related to the symptom development in the seedlings of Japanese black pine (Pinus thunbergii) families selected as resistant to pine wilt. J Jpn For Soc 89:241-248

Kusumoto D, Yonemichi T, Inoue H, Hirao T, Watanabe A, Yamada A (2014) Comparison of histological responses and tissue damage expansion between resistant and susceptible Pinus thunbergii infected with pine wood nematode Bursaphelenchus xylophilus. J For Res 19:285-294

Likens ST, Nickerson GB (1964) Detection of certain Hop oil constituents in brewing products. Am Soc Brew Chem Proc 5:13-19

Lima AS, Mendes MD, Barbosa P, Geraldes DA, Dias LS, Mota M, Barroso JG, Pedro LG, Figueiredo AC (2010) Pinewood nematode (Bursaphelenchus xylophilus) inoculated Pinus pinaster and Pinus pinea: time course study of volatiles and enantiomeric composition. 41st international symposium on essential oils, ISEO2010, Wroclaw, p 39 (abstract)

Melakeberhan H, Webster JM (1992) The insignificance of the energy requirements of Bursaphelenchus xylophilus as a causal factor in Pinus sylvestris seedling mortality. Fundam Appl Nematol 15:179-182

Mota M, Vieira P (2008) Pine wilt disease: a worldwide threat to forest ecosystems. Springer, Heidelberg

Mota M, Braasch H, Bravo MA, Penas AC, Burgermeister W, Metge K, Sousa E (1999) First report of Bursaphelenchus xylophilus in Portugal and in Europe. Nematology 1:727-734

Nascimento FX, Hasegawa K, Mota M, Vicente CSL (2014) Bacterial role in pine wilt disease development- review and future perspectives. Environ Microbiol Rep. doi:10.1111/1758-2229. 12202

Penas AC, Bravo MA, Valadas V, Mota M (2008) Detailed morphobiometric studies of Bursaphelenchus xylophilus and characterisation of other Bursaphelenchus species (Nematoda: Parasitaphelenchidae) associated with Pinus pinaster in Portugal. J Nematode Morph Syst 10:137-163

Petersen-Silva R, Naves P, Godinho P, Sousa E, Pujade-Villar J (2014) Distribution, hosts and parasitoids of Monochamus galloprovincialis (Coleoptera: Cerambycidae) in Portugal Mainland. Silva Lusitana 22:67-82
Roriz M, Santos C, Vasconcelos MW (2011) Population dynamics of bacteria associated with different strains of the pine wood nematode Bursaphelenchus xylophilus after inoculation in maritime pine (Pinus pinaster). Exp Parasitol 128:357-364

Ryss AY (2003) Express technique to prepare permanent collection slides of nematodes. Zoosyst Rossica 11:257-260

Schenk UR, Hildebrandt AC (1972) Medium and techniques for induction and growth of monocotyledonous and dicotyledonous plant cell cultures. Can J Bot 50:199-204

Shinya R, Morisaka H, Kikuchi T, Takeuchi Y, Futai K, Ueda M (2013a) Making headway in understanding pine wilt disease: what do we perceive in the postgenomic era? J Biosci Bioeng $116: 1-8$

Shinya R, Morisaka H, Takeuchi Y, Ueda M, Futai K (2013b) Secretome analysis of the pine wood nematode Bursaphelenchus xylophilus reveals the tangled roots of parasitism and its potential for molecular mimicry. PLoS ONE 8:e67377

Stamps WT, Linit MJ (2001) Interaction of intrinsic and extrinsic chemical cues in the behaviour of Bursaphelenchus xylophilus (Aphelenchida: Aphelenchoididae) in relation to its beetle vectors. Nematology 3:295-301

Takeuchi Y, Kanzaki N, Futai K (2006) Volatile compounds in pine stands suffering from pine wilt disease: qualitative and quantitative evaluation. Nematology 8:869-879

Tereso S, Gonçalves S, Marum L, Oliveira M, Maroco J, Miguel C (2006) Improved axillary and adventitious bud regeneration from Portuguese genotypes of Pinus pinaster Ait. Propag Ornam Plants 6:24-33

Umebayashi T, Fukuda K, Haishi T, Sotooka R, Zuhair S, Otsuki K (2011) The developmental process of xylem embolisms in pine wilt disease monitored by multipoint imaging using compact magnetic resonance imaging. J Exp Bot 59:3371-3381

Vicente CSL, Nascimento F, Espada M, Barbosa P, Mota M, Glick BR, Oliveira S (2012) Characterization of bacteria associated with pinewood nematode Bursaphelenchus xylophilus. PLoS ONE 7:e46661. doi:10.1371/journal.pone.0046661

Wang Z, Wang CY, Fang ZM, Zhang DL, Liu L, Lee MR, Li Z, Li JJ, Sung CK (2010) Advances in research of pathogenic mechanism of pine wilt disease. Afr J Microbiol Res 4:437-442

Washer J, Reilly KJ, Barnett JR (1977) Differentiation in Pinus radiata callus culture: the effect of nutrients. J For Sci 7:321-328

Xie LQ, Zhao BG (2008) Post-inoculation population dynamics of Bursaphelenchus xylophilus and associated bacteria in Pine Wilt Disease on Pinus thunbergii. J Phytopathol 156:385-389

Zhao B, Liu Y, Lin F (2006) Mutual influences in growth and reproduction between pine wood nematode Bursaphelenchus xylophilus and bacteria it carries. Front For China 1:324-327

Zhao L, Wei W, Kang L, Sun JH (2007) Chemotaxis of the pinewood nematode, Bursaphelenchus xylophilus, to volatiles associated with host pine, Pinus massoniana, and its vector Monochamus alternatus. J Chem Ecol 33:1207-1216

Zhao L, Mota M, Vieira P, Butcher RA, Sun J (2014) Interspecific communication between pinewood nematode, its insect vector, and associated microbes. Trends Parasitol 30:299-308

Zhu LH, Ye J, Negi S, Xu XL, Wang ZL, Ji JY (2012) Pathogenicity of aseptic Bursaphelenchus xylophilus. PLoS ONE 7:e38095. doi:10.1371/journal.pone.0038095 